\title{
Risk Profile of Ischemic Stroke
} Caused by Small-Artery Occlusion
vs. Deep Intracerebral Hemorrhage

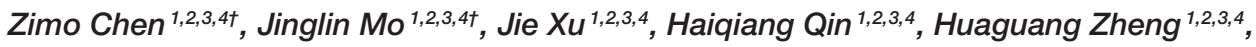 \\ Yuesong Pan 1,2,3,4, Xia Meng 1,2,3,4, Jing Jing ${ }^{1,2,3,4}$, Xianglong Xiang ${ }^{2,3,4}$ and \\ Yongjun Wang ${ }^{1,2,3,4 *}$
}

${ }^{1}$ Department of Neurology, Beijing Tiantan Hospital, Capital Medical University, Beijing, China, ${ }^{2}$ China National Clinical Research Center for Neurological Diseases, Beijing, China, ${ }^{3}$ Center of Stroke, Beijing Institute for Brain Disorders, Beijing, China, ${ }^{4}$ Beijing Key Laboratory of Translational Medicine for Cerebrovascular Disease, Beijing, China

\section{OPEN ACCESS}

Edited by:

Andreas Charidimou,

Massachusetts General Hospital,

Harvard Medical School,

United States

Reviewed by:

Aristeidis H. Katsanos,

McMaster University, Canada

Vasileios-Arsenios Lioutas,

Beth Israel Deaconess Medical

Center, Harvard Medical School,

United States

*Correspondence:

Yongjun Wang

yongjunwang@ncrcnd.org.cn

tThese authors have contributed equally to this work

Specialty section:

This article was submitted to

Stroke,

a section of the journal

Frontiers in Neurology

Received: 18 August 2019 Accepted: 30 October 2019 Published: 27 November 2019

Citation:

Chen Z, Mo J, Xu J, Qin H, Zheng H,

Pan $Y$, Meng $X$, Jing J, Xiang $X$ and Wang $Y$ (2019) Risk Profile of Ischemic

Stroke Caused by Small-Artery

Occlusion vs. Deep Intracerebral Hemorrhage. Front. Neurol. 10:1213.

doi: 10.3389/fneur.2019.01213
Background: Small-artery occlusion (SAO) subtype accounts for a quarter of the cases of ischemic stroke and is mainly caused by pathological changes in cerebral small vessels, which also involve in deep intracerebral hemorrhage $(\mathrm{dl} C \mathrm{CH})$. However, the factors that drive some cases to $\mathrm{SAO}$ and others to $\mathrm{dICH}$ remained incompletely defined.

Material and Methods: This study is a cross-sectional study from the China National Stroke Registry that included consecutive patients with ischemic stroke or intracerebral hemorrhage between August 2007 and September 2008. We compared the risk profile between the two subgroups using multivariable logistic regression.

Results: A total of 1,135 patients with SAO stroke and 1,125 $\mathrm{dlCH}$ patients were included for analyses. Generally, patients with SAO stroke were more likely to be male (odds ratio $=0.74$, confidence interval $=0.58-0.94)$ and have diabetes $(0.30$, 0.22-0.40), higher atherogenic lipid profiles, higher body mass index $(0.96,0.94-0.99)$, higher waist/height ratio $(0.12,0.03-0.48)$, higher platelet count $(0.84,0.77-0.91)$, and higher proportion of abnormal estimated glomerular filtration rate $(<90, \mathrm{ml} / \mathrm{min} / 1.73$ $\left.\mathrm{m}^{2}\right)(0.77,0.62-0.95)$. Conversely, patients with $\mathrm{dICH}$ were more likely to have higher blood pressure parameters, inflammation levels (white blood cell count: 1.61, 1.48-1.76; high sensitivity C-reactive protein: 2.07, 1.36-3.16), and high-density lipoprotein-C (1.57, 1.25-1.98).

Conclusions: The risk profile between SAO stroke and $\mathrm{dICH}$ were different. Furthermore, despite of traditional indexes, waist/height ratio, platelet count, inflammation levels, lipid profile, and estimated glomerular filtration rate also play important roles in driving arteriolosclerosis into opposite ends.

Keywords: cerebrovascular disease, lacunar stroke, intracerebral hemorrhage, risk factor, inflammation

\section{INTRODUCTION}

Small-artery occlusion (SAO), a distinct ischemic stroke (IS) subtype resulting in small ( $<15 \mathrm{~mm}$ in axial diameter) subcortical infarcts, is thought to correlate with intrinsic disorders of perforating cerebral arterioles called arteriolosclerosis (1), due to multiple risk factors, including age, hypertension, and diabetes (2). Intracranial hemorrhage (ICH) can be classified into deep intracerebral hemorrhage $(\mathrm{dICH})$ and lobar $\mathrm{ICH}$, according to the location of the lesion. $\mathrm{dICH}$ 
is more likely related to longstanding hypertension (3), which results in hypertensive vasculopathy and causes microscopic degenerative changes in the wall of small-to-medium penetrating vessels (4), while lobar ICH is multimorbid and widely known as cerebral amyloid angiopathy (CAA) related disease $(5,6)$.

However, SAO stroke and $\mathrm{dICH}$ represent opposite ends of the similar pathological process in cerebral small vessels. It was shown that patients with SAO were older and more likely to have diabetes mellitus and higher cholesterol level, while $\mathrm{dICH}$ patients tended to be excessive alcohol consumers and have hypertension $(7,8)$. However, controversy still remained on other risk factors for cerebrovascular disease. It was demonstrated that renal dysfunction led to the thickening of vascular wall in arteriosclerosis (9). Moreover, previous studies have verified that platelet (PLT) also actively participate in the pathology of arteriosclerosis (10). Besides, recent findings provided strong evidence for the inflammatory hypothesis of atherosclerosis (11, 12). However, to date, no evidence has shown the above factors in differentiating SAO stroke and $\mathrm{dICH}$.

Therefore, this investigation focused on more detailed differences and points of controversy between the two groups.

\section{MATERIALS AND METHODS \\ Study Cohort and Population}

This is a cross-sectional study from the China National Stroke Registry (CNSR), which consecutively enrolled stroke patients ( $\geq 18$ years) within 14 days after stroke onset from 132 participating hospitals in China. Stroke was defined as acute IS, intracerebral hemorrhage, and subarachnoid hemorrhage according to the World Health Organization criteria. The diagnosis was confirmed by brain computed tomography (CT) or magnetic resonance imaging (MRI) (13). The participating hospitals covered 100 tertiary and 32 secondary urban hospitals from 27 provinces and 4 municipalities in China, including the Hong Kong region, between September 2007 and August 2008. Written informed consent was issued by all participants. The study was approved by the Central Institutional Review Board at Beijing Tiantan Hospital. Demographic information (age, sex), medical variables [admission systolic blood pressure (SBP), diastolic blood pressure (DBP), pulse pressure (PP), and mean arterial pressure (MAP), reported history of hypertension, history of diabetes mellitus, history of coronary heart disease, history of dyslipidemia, history of anticoagulant drug use, and history of alcohol or tobacco use], and laboratory studies on admission [estimated glomerular filtration rate (eGFR), PLT count, white blood cell (WBC) count, high sensitivity C-reactive protein (hs-CRP) level, triglyceride (TG) level, total cholesterol (TC) level, low-density lipoprotein cholesterol (LDL-c) level, high-density lipoprotein cholesterol (HDL-c) level, waist/height ratio (WHtR), and body mass index (BMI)] were documented in paper-based case report forms.

\section{Defining $\mathrm{dICH}$ and SAO}

The diagnostic criteria for SAO were defined as follows: patients who had one of the traditional clinical lacunar syndromes (including pure motor stroke, pure sensorimotor stroke, pure sensory stroke, ataxic hemiparesis, or clumsy hand dysarthria) along with brain imaging (CT or MRI) findings (infarction diameter $<1.5 \mathrm{~cm}$ in the appropriate region) and did not show evidence of cerebral cortical dysfunction. Evidence of cardiac sources of embolism was absent, and large extracranial arteries showed a stenosis of $<50 \%$ in an ipsilateral artery (14). ICH was defined according to World Health Organization criteria (13), and $\mathrm{dICH}$ was located at the following regions: putamen, caudate nucleus, internal capsule, thalamus, and brain stem. Cortical hemorrhage, deep intracerebral hemorrhage, intraventricular hemorrhage, prestroke modified Ranking Scale $>2$, lack of data for hematoma volume, and hemorrhage resulting from trauma, underlying tumor, aneurysm, or arteriovenous malformation were excluded (15).

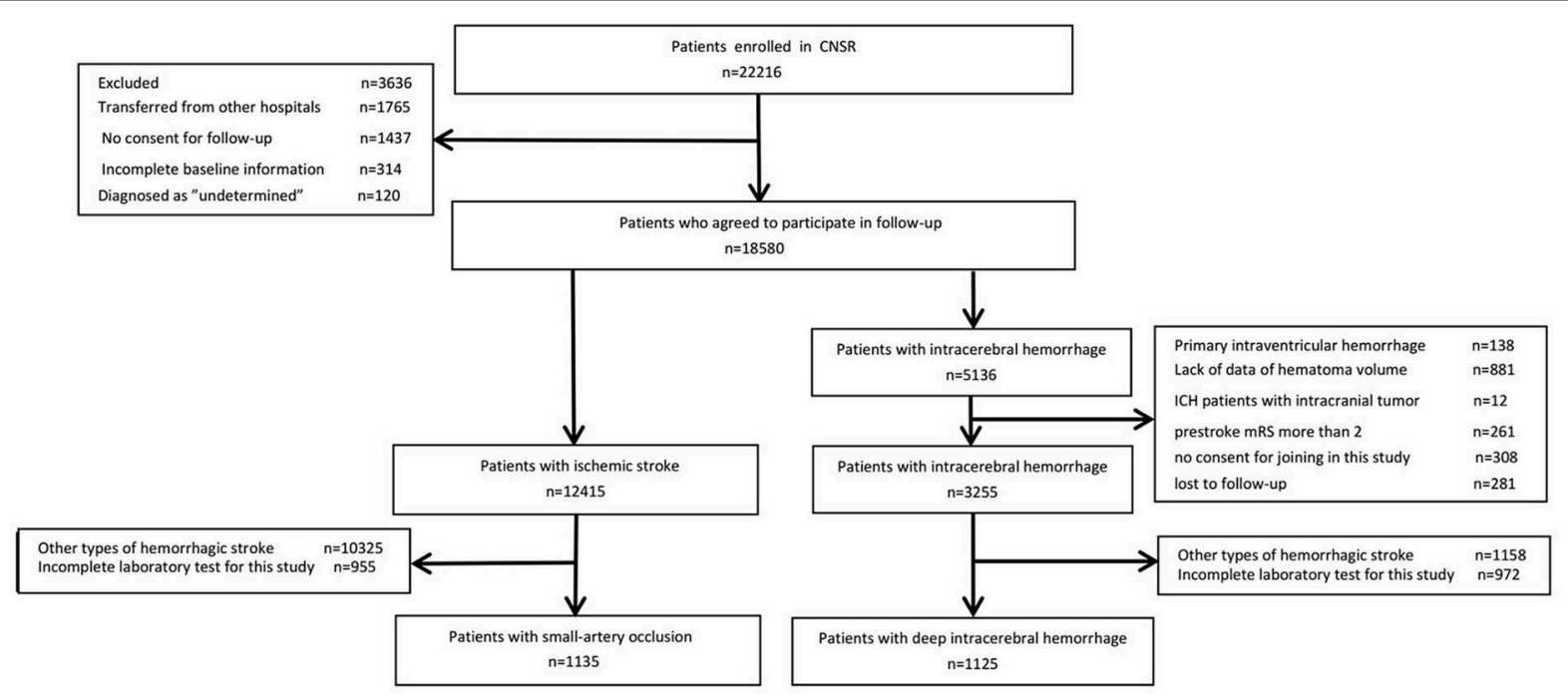

FIGURE 1 | Patient flow diagram. CNSR, China National Stroke Registry; ICH, intracranial hemorrhage; mRS, modified Ranking Scale. 


\section{Risk Factor Definition}

Hypertension was defined by SBP $\geq 140 \mathrm{mmHg}$ and/or DBP $\geq$ $90 \mathrm{mmHg}$ out of the acute phase or the use of pharmacological treatment for hypertension. PP was calculated as SBP-DBP, and MAP was calculated as DBP +0.33 (SBP-DBP). Other risk factors were defined as follows: dyslipidemia (TC level $\geq 240$ $\mathrm{mg} / \mathrm{dl}$, HDL-c level $<35 \mathrm{mg} / \mathrm{dl}$, or the use of lipid-lowering agents), diabetes mellitus (fasting blood glucose level $\geq 120$ $\mathrm{mg} / \mathrm{dl}$ or current treatment with antidiabetic drugs), current or previous smoker (an individual who smoked at the time of stroke or had quit smoking within 1 year), or heavy alcohol consumption ( $\geq 5$ standard alcoholic beverages per day). We also collected information on coronary artery disease (medical history of angina, myocardial infarction, coronary artery bypass graft, or percutaneous transluminal coronary angioplasty), and treatment with anticoagulant drugs (previously documented in medical files). The details and definitions have been described previously (16).

Data were obtained from interviews with patients, next of kin, and/or attending physicians or general practitioners. Fasting lipid levels, WBC count, hs-CRP, PLT count, and eGFR measurements

TABLE 1 | Characteristics of the study population.

\begin{tabular}{|c|c|}
\hline Characteristics & \\
\hline Age, mean (SD), year & $63.0(12.1)$ \\
\hline Sex, male, $n(\%)$ & $1,436(63.5)$ \\
\hline History of hypertension, $n(\%)$ & $1,546(68.4)$ \\
\hline Admission SBP, mean (SD), mmHg & $158.4(26)$ \\
\hline Admission DBP, mean (SD), mmHg & $92.5(15.5)$ \\
\hline Admission PP, mean (SD), mmHg & $65.8(19.5)$ \\
\hline Admission MAP, mean (SD), mmHg & $114.5(17.3)$ \\
\hline History of diabetes mellitus, $n$ (\%) & $365(16.2)$ \\
\hline History of coronary heart disease, $n(\%)$ & $38(1.7)$ \\
\hline History of anticoagulant drug, $n(\%)$ & $26(1.2)$ \\
\hline Current smoker, $n(\%)$ & $948(42.0)$ \\
\hline Heavy alcohol consumption, $n(\%)$ & $79(3.5)$ \\
\hline History of dyslipidemia, $n$ (\%) & $225(10.0)$ \\
\hline Admission LDL-c, median (IQR), mmol// & $2.7(2.1-3.3)$ \\
\hline Admission TG, median (IQR), mmol// & $1.4(1.0-2.0)$ \\
\hline Admission TC, median (IQR), mmol// & $4.6(3.9-5.3)$ \\
\hline Admission HDL-c, median (IQR), mmol/l & $1.2(1.0-1.4)$ \\
\hline AIP, median (IQR) & $0.1-0.1-0.3)$ \\
\hline non-HDL-C, median (IQR) & $3.3(2.7-4.0)$ \\
\hline LDL-c/HDL-c, median (IQR) & $2.2(1.7-2.9)$ \\
\hline TC/HDL-c, median (IQR) & $3.8(3.1-4.71)$ \\
\hline $\mathrm{LCl}$, median (IQR) & $13.8(7.4-25.6)$ \\
\hline Al, median (IQR) & $2.8(2.1-3.7)$ \\
\hline $\mathrm{BMl}$, mean (SD), $\mathrm{kg} / \mathrm{m}^{2}$ & $24.3(3.9)$ \\
\hline WHtR, mean (SD) & $0.5(0.1)$ \\
\hline PLT count, median (IQR) & $190(149-232)$ \\
\hline WBC count, median (IQR), 109/I & $7.5(6.1-9.5)$ \\
\hline eGFR, median (IQR), ml/min/1.73 m² & $83.5(67.6-96.8)$ \\
\hline
\end{tabular}

$S B P$, systolic blood pressure; $D B P$, diastolic blood pressure; $P P$, pulse pressure; MAP, mean arterial pressure; TG, triglyceride; TC, total cholesterol; LDL-C, low-density lipoprotein cholesterol; HDL-c, high-density lipoprotein cholesterol; AIP, atherogenic index of plasma; $\mathrm{LCl}$, lipoprotein combine index; $\mathrm{Al}$, atherogenic index; $\mathrm{BMI}$, body mass index; WHtR, waist/height ratio; PLT count, platelet count; WBC count, white blood cell count; eGFR, estimated glomerular filtration rate; IQR, interquartile range; $S D$, standard deviation. were carried out on venous blood samples obtained within $24 \mathrm{~h}$ of stroke occurrence in each participating center, using comparable procedures. eGFR was calculated using the Chronic Kidney Disease Epidemiology Collaboration creatinine equation with an adjusted coefficient of 1.1 for the Asian population $(17,18)$. BMI was calculated by dividing body weight in kilograms by the square of body length in meters $\left(\mathrm{kg} / \mathrm{m}^{2}\right)$. WHtR was obtained by dividing the waist circumference $(\mathrm{cm})$ by height $(\mathrm{cm})$. The atherogenic index of plasma (AIP) is defined as the base 10 logarithm of the ratio of the concentration of TG to HDL-c; the non-HDL-c is defined as TC minus HDL-c; the atherogenic index (AI) is defined as the ratio of non-HDL-c to HDL-c; and the lipoprotein combine index (LCI) is defined as the ratio of TC*TG*LDL-c to HDL-c (19).

\section{Statistical Analysis}

Parametric continuous variables were analyzed by the $t$-test, and results were presented as means with SD. Non-parametric variables were analyzed with the Wilcoxon rank-sum test, and results are presented as medians with interquartile range. Categorical variables are presented as proportions $(n \%)$, and intergroup differences were tested with the $\chi^{2}$ test. Multivariable logistic regression was performed including demographic factors (age and sex) as well as a history of hypertension, SBP, DBP, history of diabetes mellitus, history of coronary heart disease, history of hypercholesterolemia, TG, TC, LDL-c, HDL-c, AIP, non-HDL-c, TC/HDL-c, LDL-c/HDL-c, AI, LCI, current or previous smoker, heavy alcohol consumption, BMI, WHtR, eGFR, PLT count, hs-CRP, and WBC count as covariates. SAO group is the reference group in evaluating odds ratios. The covariates above were analyzed by several models to avoid the selected factors concurring with each other. In model of pressure values (SBP, DBP, PP, and MAP), covariates included age, sex, history of coronary heart disease, current or previous smoker, heavy alcohol consumption, history of diabetes mellitus, TG, TC, HDL-c, BMI, PLT count, WBC count, and eGFR. In the model of traditional and nontraditional lipid profiles, covariates included age, sex, SBP, DBP, history of coronary heart disease, current or previous smoker, heavy alcohol consumption, history of diabetes mellitus, TG, TC, HDL-c, BMI, PLT count, WBC count, and eGFR. In the model of CRP, covariates included age, sex, SBP, DBP, history of coronary heart disease, current or previous smoker, heavy alcohol consumption, history of diabetes mellitus, TC, BMI, and eGFR. $P<0.05$ on a two-sided test was considered as significant. Data were analyzed using SAS 9.4.

\section{RESULTS}

Of the 22,216 patients enrolled in the China National Stroke Registry, 18,580 patients had complete baseline information and agreed to participate in follow-up. Among them, 12,415 had IS and 5,136 had ICH. For IS patients, 955 without complete data of laboratory test and 10,325 for other types of IS were further excluded, leaving 1,135 SAO stroke patients included in the final analysis. For patients with $\mathrm{ICH}$, after exclusion for other types of ICH, incomplete data of laboratory test and other reasons, $1,125 \mathrm{dICH}$ patients were included for the comparison with SAO stroke (Figure 1). 
TABLE 2 | Univariable analysis between SAO stroke and dICH.

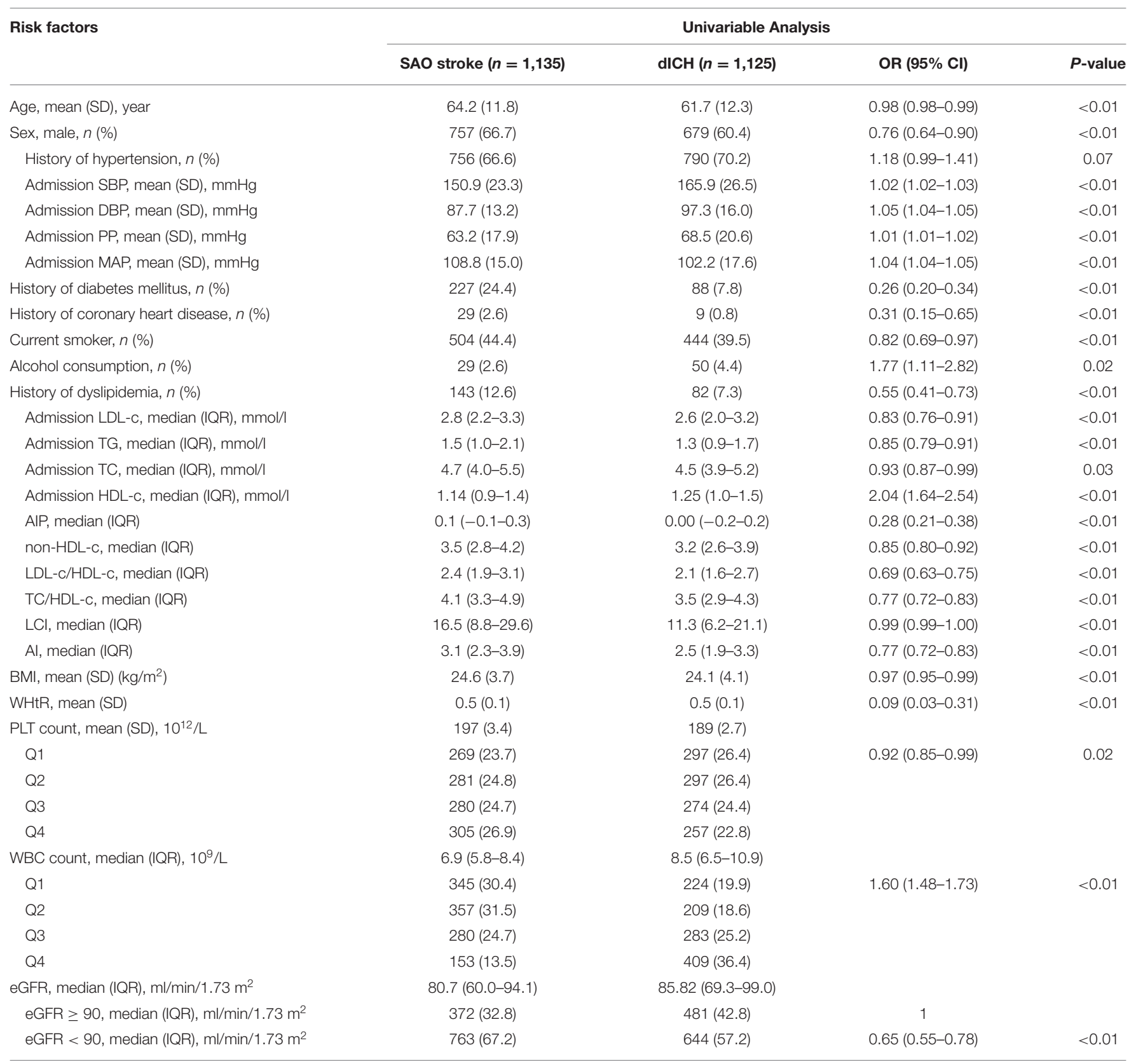

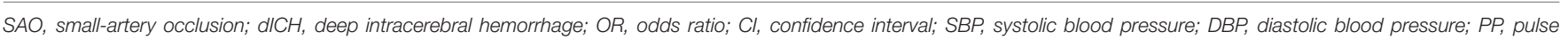

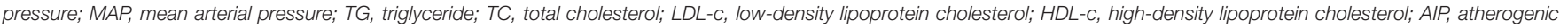

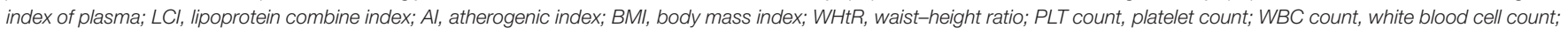
eGFR, estimated glomerular filtration rate; IQR, interquartile range; $S D$, standard deviation.

The average age of the included patients was $63.0 \pm 12.1$ years (male, 63.54\%). Descriptive statistics of the whole study group are summarized in Table 1. Univariable and multivariable logistic regression analyses were performed to determine the association of risk factors with stroke type (Tables 2, 3). In univariable analysis (Table 2), patients with SAO were more likely to be older, male, to smoke, to have history of diabetes, coronary heart disease, and dyslipidemia, to have higher TG, TC, LDL-c, BMI,
WHtR, and PLT count and lower eGFR. Patients with dICH were more likely to be heavy alcohol consumers and have higher SBP, DBP, PP, MAP, HDL-c WBC count, and hs-CRP.

We further performed several models of multivariable analyses in case of the factors concurring with each other. In the model adjusting medical history of hypertension, diabetes mellitus, and dyslipidemia, age, smoking, and heavy alcohol consumption were not consistently significant (Table S1). In the 
TABLE 3 | Multivariable regression analysis of indexes between SAO stroke and $\mathrm{dICH}$.

\begin{tabular}{lcc}
\hline Risk factors & $\begin{array}{c}\text { Multivariable Analysis } \\
\text { OR (95\% Cl) }\end{array}$ & P-value \\
& $0.99(0.98-1.00)$ & 0.06 \\
Age & $0.74(0.58-0.94)$ & 0.01 \\
Sex, male & $1.01(1.01-1.02)$ & $<0.01$ \\
Admission SBP & $1.03(1.02-1.04)$ & $<0.01$ \\
Admission DBP & $0.57(0.25-1.33)$ & 0.19 \\
History of coronary heart disease & $0.74(0.59-0.94)$ & 0.01 \\
Current or previous smoker & $1.52(0.89-2.58)$ & 0.12 \\
Heavy alcohol consumption & $0.30(0.22-0.40)$ & $<0.01$ \\
History of diabetes mellitus & $0.89(0.82-0.96)$ & $<0.01$ \\
Admission TG & $0.89(0.82-0.97)$ & $<0.01$ \\
Admission TC & $1.66(1.30-2.13)$ & $<0.01$ \\
Admission HDL-c & $0.96(0.94-0.99)$ & $<0.01$ \\
BMl & $0.84(0.77-0.91)$ & $<0.01$ \\
PLT count & $1.61(1.48-1.76)$ & $<0.01$ \\
WBC count & -1.00 & 0.02 \\
eGFR & $0.77(0.62-0.95)$ & \\
eGFR $\geq 90 \mathrm{ml} / \mathrm{min} / 1.73 \mathrm{~m}^{2}$ & - & \\
eGFR $<90 \mathrm{ml} / \mathrm{min} / 1.73 \mathrm{~m}^{2}$ & & \\
\hline
\end{tabular}

SAO, small-artery occlusion; dICH, deep intracerebral hemorrhage; OR, odds ratio; $\mathrm{Cl}$, confidence interval; SBP, systolic blood pressure; DBP, diastolic blood pressure; TG, triglyceride; TC, total cholesterol; HDL-c, high-density lipoprotein cholesterol; BMI, body mass index; PLT quartiles, platelet quartiles; WBC quartiles, white blood cell quartiles; eGFR, estimated glomerular filtration rate.

model adjusting indexes of pressure and serum lipid instead of the medical histories, compared with univariable analysis, age, history of coronary heart disease, and heavy alcohol consumption were not significant (Table 3). Multivariable models for MAP and PP showed that higher MAP and PP were significantly related to $\mathrm{dICH}$ in accord with SBP and DBP (Tables S2, S3). Moreover, in the multivariable model for WHtR instead of BMI, higher WHtR was associated with SAO, which favored the result of BMI (Table S4).

In multivariable analysis models for traditional and nontraditional lipid profile, all results indicated that SAO patients are likely to have higher level of atherogenic lipid profile such as higher LDL-c, TC, and TG, as well as other non-traditional lipid profile such as AIP and lower HDL-c (Figure 2).

On the contrary, higher inflammation levels were associated with higher possibility of $\mathrm{dICH}$. Our results showed that dICH patients have higher WBC count than SAO patients. To consolidate the conclusion, we further performed the subgroup analysis including 449 patients with available value of hs-CRP. Consistently, we found that higher hs-CRP level was associated with dICH (Table S5).

\section{DISCUSSION}

The main finding of our study is that SAO stroke and $\mathrm{dICH}$ exhibit different risk profiles although they share similar pathophysiological process of cerebral small vessels. In comparison with previous studies, we included more detailed patient characteristics and found their significant roles in differentiating SAO stroke and $\mathrm{dICH}$, such as traditional and non-traditional lipid profiles, inflammation levels, PLT count, BMI, WHtR, WBC count, hs-CRP, and eGFR in our study, which were rarely analyzed before. Thus, our work provides a more comprehensive view on the impact of risk factors in driving arteriolosclerosis into opposite ends.

In our study, history of hypertension and the specific pressure values (SBP, DBP, PP, and MAP) played a dominant role in $\mathrm{dICH}$, which favored previous findings $(7,8)$. Contrary to hypertension, diabetes mellitus was associated with SAO stroke, which was also consistent with previous reports (19-21). Furthermore, we observed that elevated PLT count were more strongly associated with SAO stroke. Platelets play a key role in the development of IS via their role in evolution of atherosclerosis (22). In contrast, thrombocytopenia has been found to be associated with spontaneous intracerebral hemorrhage (23). In addition, PLT count have also been recorded as an important index for development and prognosis of ischemic and hemorrhagic stroke $(24,25)$.

Investigations remain limited by far on BMI or WHtR between SAO and dICH. In accordance with previous studies (26-29), our findings agreed that lower BMI and WHtR were more relevant to $\mathrm{dICH}$. We hypothesize that the adipose tissue may play an important role in shifting the cerebral small vessel disease manifestations toward either ischemia or hemorrhage (28). Furthermore, we compared the role of various blood lipid parameters including traditional (TG, TC, LDL$c$, and HDL-c) and non-traditional lipid profiles (AIP, nonHDL-c, TC/HDL-c, LDL/HDL-c, AI, and LCI) between dICH and SAO stroke. Numerous studies focused on the association between lipid profiles and stroke subtypes but yielded conflict results. The China Kadoorie Biobank study with 512,891 participants found that plasma concentrations of LDL-C and TG were positively associated with risk of IS and inversely associated with a risk of ICH. Moreover, plasma concentrations of HDL-C were inversely associated with risk of IS, but not with ICH. Furthermore, the causal relevance of LDL-C for both IS and ICH was confirmed by Mendelian randomization analyses in this study (30). However, two studies enrolled elderly adults and observed no association between LDL-C levels and the risk of ICH $(31,32)$. In Women's Health Study with 27,937 women enrolled, they reported that low LDL$\mathrm{C}$ levels and low TG levels were associated with increased risk of hemorrhagic stroke, but no association between HDL$\mathrm{C}$ levels and the risk of $\mathrm{ICH}$ was found. Most lipid profiles analyzed in our study were shown to significantly participate in differentiating SAO from $\operatorname{dICH}(33,34)$. The analyses demonstrated that ICH patients tend to have lower level of atherogenic lipid profiles. It is supported by the histopathological studies demonstrating that lower cholesterol concentrations may increase permeability of the vessel walls $(35,36)$, causing arterionecrosis and microaneurysms, which is often found in ICH. AIP was shown to be a better marker to reflect increased cardiovascular disease risk than TC, LDL-c, HDL-c, and other nontraditional lipid profiles (37). Our results also showed 


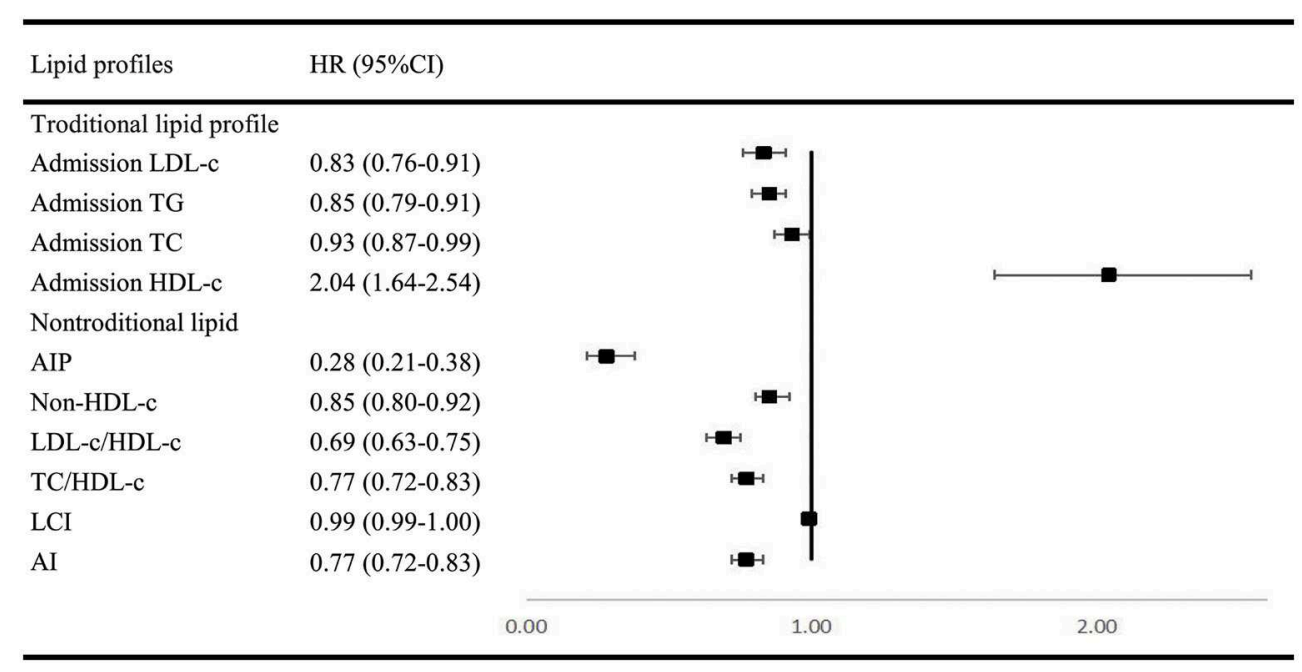

FIGURE 2 | Multivariable regression analysis of lipid profile between SAO stroke and dICH. SAO, small-artery occlusion; dICH, deep intracerebral hemorrhage; OR, odds ratio; CI, confidence interval; TG, triglyceride; TC, total cholesterol; LDL-c, low-density lipoprotein cholesterol; HDL-c, high-density lipoprotein cholesterol; AIP, atherogenic index of plasma; LCl, lipoprotein combine index; Al, atherogenic index.

that AIP may have a better capability in distinguishing SAO from dICH.

Evidence suggested that inflammation played a key role in the pathogenesis of cardiovascular diseases (38), and increased inflammation levels were related to the severity, disability, and mortality of both ischemic and hemorrhagic stroke (39). However, it was rarely considered in analysis as a differential characteristic between $\mathrm{dICH}$ and SAO stroke. Previous studies have shown that inflammatory cytokines were involved in the pathogenesis of cerebral small vessel disease and differing inflammatory pathways between ischemic and hemorrhagic manifestations of cerebral small vessel disease (40). As markers of inflammation, the increase in WBC count and hs-CRP was significantly associated with $\mathrm{dICH}$ compared with SAO group in our investigation. Thus, we proposed that the intensive systematic inflammation may involve in the weakening of vessel wall and lead to vessel rupture. However, due to the design of this cross-sectional study, it remains unknown whether the observed higher level of hs-CRP in ICH patients results from the inflammation or is just an acute phase reactant.

Our study has several limitations: First, the lack of MRI data about the presence/absence of neuroradiological markers of small vessel disease prevented us from further revealing the differences such as white matter hyperintensities and microbleed between SAO stroke and $\mathrm{dICH}$, and may cause the confounders for selection of cases for the present study. Second, our study is a cross-sectional study, but the indexes such as blood pressure, lipid values, and WBC count were collected at the acute phase of stroke, which cannot fully reflect the long-term behavior. Third, because of the lack of a stroke-free control group, we cannot conclude but speculate that the observed associations might represent the causality in a way, and further relationships need to be investigated in the future. Fourth, the lack of centralization for biological measures and radiological data may also cause the potential biases. Fifth, our study population is based on Asian population; therefore, the findings may not be generalized directly to all populations. Our study needs to be investigated in a longitudinal prospective cohort to further reveal the association between risk factors and different subtype of stroke occurrence. In addition, elucidating the differences in neuroradiological markers of small vessel disease by MRI is needed to deeply demonstrate the underlying mechanism between $\mathrm{SAO}$ and $\mathrm{dICH}$ in future investigation.

\section{CONCLUSIONS}

On the basis of the traditional risk profiles, this study adds evidence that higher PLT count, higher level of atherogenic lipid profile, and abnormal eGFR were in favor of SAO stroke as compared to dICH. Patients with $\mathrm{dICH}$ were more likely to have higher inflammation levels and higher HDL-c.

\section{DATA AVAILABILITY STATEMENT}

The datasets generated for this study are available on request to the corresponding author.

\section{ETHICS STATEMENT}

The studies involving human participants were reviewed and approved by the ethics committee of Beijing Tiantan Hospital. The patients/participants provided their written informed consent to participate in this study. 


\section{AUTHOR CONTRIBUTIONS}

YW contributed to the conception and design of the study. ZC and JM contributed to manuscript drafting and critical revisions of the manuscript. JX, HQ, HZ, XM, and JJ contributed to the acquisition and analysis of data. YP and $\mathrm{XX}$ contributed to statistical analysis.

\section{FUNDING}

The China National Stroke Registry was funded by the Ministry of Sciences and Technology and the Ministry of Health of the People's Republic of China (Grant nos. 2006BA101A11, 2009CB521905, and 200902004) and the National Science Foundation (Grant no. 81071115). The current analysis of the CNSR data were supported by Beijing Novartis Pharma

\section{REFERENCES}

1. Fisher CM. Lacunar strokes and infarcts. A review. Neurology. (1982) 32:8716. doi: 10.1212/WNL.32.8.871

2. Wardlaw JM, Smith C, Dichgans M. Mechanisms of sporadic cerebral small vessel disease: insights from neuroimaging. Lancet Neurol. (2013) 12:483-97. doi: 10.1016/S1474-4422(13)70060-7

3. Ariesen MJ, Claus SP, Rinkel GJE, Algra A. Risk factors for intracerebral hemorrhage in the general population. a systematic review. Stroke. (2003) 34:2060-5. doi: 10.1161/01.STR.0000080678.09344.8D

4. Fisher CM. Pathological observations in hypertensive cerebral hemorrhage. J Neuropathol Exp Neurol. (1971) 30:536. doi: 10.1097/00005072-197107000-00015

5. Matsukawa H, Shinoda M, Fujii M, Takahashi O, Yamamoto D, Murakata A, et al. Factors associated with lobar vs. non-lobar intracerebral hemorrhage. Acta Neurol Scand. (2012) 126:116-21. doi: 10.1111/j.1600-0404.2011.01615.x

6. Rodrigues MA, Samarasekera N, Lerpiniere C, Humphreys C, McCarron MO, White PM, et al. The Edinburgh CT and genetic diagnostic criteria for lobar intracerebral haemorrhage associated with cerebral amyloid angiopathy: model development and diagnostic test accuracy study. Lancet Neurol. (2018) 17:232-40. doi: 10.1016/S1474-4422(18)30006-1

7. Morotti A, Paciaroni M, Zini A, Silvestrelli G, Del Zotto E, Caso V, et al. Risk profile of symptomatic lacunar stroke versus nonlobar intracerebral hemorrhage. Stroke. (2016) 47:2141-3. doi: 10.1161/STROKEAHA.116.013722

8. Labovitz DL, Bodenalbala B, Hauser WA, Sacco RL. Lacunar infarct or deep intracerebral hemorrhage. Neurology. (2007) 68:606-8. doi: 10.1212/01.wnl.0000254619.98089.43

9. Campean V, Neureiter D, Varga I, Runk F, Reiman A, Garlichs C, et al. Atherosclerosis and vascular calcification in chronic renal failure. Kidney Blood Press Res. (2005) 28:280-9. doi: 10.1159/000090182

10. Linden $\mathrm{MD}$, Jackson $\mathrm{DE}$, Platelets: pleiotropic roles in atherogenesis and atherothrombosis. Int J Biochem Cell Biol. (2010) 42:1762-6. doi: 10.1016/j.biocel.2010.07.012

11. Ridker PM, MacFadyen JG, Everett BM, Libby P, Thuren T, Glynn RJ, CANTOS Trial Group. Relationship of C-reactive protein reduction to cardiovascular event reduction following treatment with canakinumab: a secondary analysis from the CANTOS randomised controlled trial. Lancet. (2018) 391:319-28.

12. Hansson GK, Libby P. The immune response in atherosclerosis: a doubleedged sword. Nat Rev Immunol. (2006) 6:508-19. doi: 10.1038/nri1882

13. Stroke-1989. Recommendations on stroke prevention, diagnosis, and therapy. Report of the WHO Task Force on Stroke and other Cerebrovascular Disorders. Stroke. (1989) 20:1407. doi: 10.1161/01.STR.20. 10.1407
Co, Ltd. In compliance with the Uniform Requirements, for manuscripts, established by the International Committee of Medical Journal Editors, Novartis did not impose any impediment, directly or indirectly, on the publication of the study's results.

\section{ACKNOWLEDGMENTS}

We would like to convey our appreciation to all patients who participated in this study.

\section{SUPPLEMENTARY MATERIAL}

The Supplementary Material for this article can be found online at: https://www.frontiersin.org/articles/10.3389/fneur. 2019.01213/full\#supplementary-material

14. Adams HP Jr, Bendixen BH, Kappelle LJ, Biller J, Love BB, Gordon DL, et al. Classification of subtype of acute ischemic stroke. Definitions for use in a multicenter clinical trial. TOAST. Trial of Org 10172 in Acute Stroke Treatment. Stroke. (1993) 24:35-41. doi: 10.1161/01.STR.24.1.35

15. Li Z, Zhao X, Wang Y, Wang C, Liu L, Shao X, et al. Association between seizures and outcomes among intracerebral hemorrhage patients: the China National Stroke Registry. J Stroke Cerebrovasc Dis. (2015) 24:455-64. doi: 10.1016/j.jstrokecerebrovasdis.2014.09.021

16. Wang W, Lu J, Wang Y, Wang C, Wang Y, Hoff K, et al. Clinical characteristics, management, and functional outcomes in Chinese patients within the first year after intracerebral hemorrhage: analysis from China National Stroke Registry. CNS Neurosci Ther. (2012) 18:773-80. doi: 10.1111/j.1755-5949.2012.00367.x

17. Teo BW, Xu H, Wang D, Li J, Sinha AK, Shuter B, et al. GFR estimating equations in a multiethnic Asian population. Am J Kidn Dis. (2011) 58:56-63. doi: 10.1053/j.ajkd.2011.02.393

18. Levey AS, de Jong PE, El Nahas M, Astor BC, Matsushita K, Gansevoort RT, et al. The definition, classification, and prognosis of chronic kidney disease: a KDIGO Controversies Conference report. Kidney Int. (2011) 80:17-28. doi: 10.1038/ki.2010.483

19. Jamrozik K, Broadhurst R, Anderson C, Stewart-Wynne E. The role of lifestyle factors in the etiology of stroke. A population-based case-control study in Perth, Western Australia. Stroke. (1994) 25:51-9. doi: 10.1161/01.STR.25.1.51

20. Andersen K, Olsen T, Dehlendorff C, Kammersgaard L. Hemorrhagic and ischemic strokes compared: stroke severity, mortality, and risk factors. Stroke. (2009) 40:2068-72. doi: 10.1161/STROKEAHA.108.540112

21. Kaplan E, Gottesman R, Llinas R, Marsh E. The Association between specific substances of abuse and subcortical intracerebral hemorrhage versus ischemic lacunar infarction. Front Neurol. (2014) 5:174. doi: 10.3389/fneur.2014.00174

22. del Zoppo GJ. The role of platelets in ischemic stroke. Neurology. (1998) 51:9-14. doi: 10.1212/WNL.51.3_Suppl_3.S9

23. Rajajee V, Brown DM, Tuhrim S. Coagulation abnormalities following primary intracerebral hemorrhage. J Stroke Cerebrovasc Dis. (2004) 13:47-51. doi: 10.1016/j.jstrokecerebrovasdis.2004.01.002

24. Maydadomaç F, Misirli H, Yilmaz M. Prognostic role of mean platelet volume and platelet count in ischemic and hemorrhagic stroke. J Stroke Cerebrovasc Dis. (2010) 19:66-72. doi: 10.1016/j.jstrokecerebrovasdis.2009.03.003

25. Du J, Wang Q, He B, Liu P, Chen J, Quan H, et al. Association of mean platelet volume and platelet count with the development and prognosis of ischemic and hemorrhagic stroke. Int J Lab Hematol. (2016) 38:233-9. doi: 10.1111/ijlh.12474

26. Kroll ME, Green J, Beral V, Sudlow CL, Brown A, Kirichek O, et al. Adiposity and ischemic and hemorrhagic stroke: prospective study in women and meta-analysis. Neurology. (2016) 87:1473-81. doi: 10.1212/WNL.0000000000003171 
27. Bazzano L, Gu D, Whelton MR, Wu X, Chen CS, Duan X, et al. Body mass index and risk of stroke among Chinese men and women. Ann Neurol. (2010) 67:11-20. doi: 10.1002/ana.21950

28. Lioutas VA, Beiser A, Himali J, Aparicio H, Romero JR, DeCarli C, et al. Lacunar infarcts and intracerebral hemorrhage differences: a nested casecontrol analysis in the FHS (Framingham Heart Study). Stroke. (2017) 48:4869. doi: 10.1161/STROKEAHA.116.014839

29. Biffi A, Cortellini L, Nearnberg CM, Ayres AM, Schwab K, Gilson AJ, et al. Body mass index and etiology of intracerebral hemorrhage. Stroke. (2011) 42:2526-30. doi: 10.1161/STROKEAHA.111.617225

30. Sun L, Clarke R, Bennett D, Guo Y, Walters RG, Hill M, et al. Causal associations of blood lipids with risk of ischemic stroke and intracerebral hemorrhage in Chinese adults. Nat Med. (2019) 25:569-74. doi: 10.1038/s41591-019-0366-x

31. Wieberdink RG, Poels MMF, Vernooij MW, Koudstaal PJ, Hofman A, van der LA,et al. Serum lipid levels and the risk of intracerebral hemorrhage: the Rotterdam Study. Arterioscler Thromb Vasc Biol. (2011) 31:2982-9. doi: 10.1161/ATVBAHA.111.234948

32. Bonaventure A, Kurth T, Pico F, Barberger-Gateau P, Ritchie K, Stapf $\mathrm{C}$, et al. Triglycerides and risk of hemorrhagic stroke vs. ischemic vascular events: the Three-City Study. Atherosclerosis. (2010) 210:243-8. doi: 10.1016/j.atherosclerosis.2009.10.043

33. Iso H, Jacobs DR, Wentworth D, Neaton JD, Cohen JD. Serum cholesterol levels and six-year mortality from stroke in 350,977 men screened for the multiple risk factor intervention trial. N Engl J Med. (1989) 320:904-10. doi: 10.1056/NEJM198904063201405

34. Puddey IB. Low serum cholesterol and the risk of cerebral hemorrhage. Atherosclerosis. (1996) 119:1-6. doi: 10.1016/0021-9150(95)05634-3

35. Ooneda G, Yoshida Y, Suzuki K, Shinkai H, Hori S, Kobori K, et al. Smooth muscle cells in the development of plasmatic arterionecrosis, arteriosclerosis and arterial contraction. Blood Vessels. (1978) 15:148-56. doi: $10.1159 / 000158160$
36. Konishi M, Iso $\mathrm{H}$, Komachi $\mathrm{Y}$, Iida M, Shimamoto T, Jacobs DR Jr, et al. Associations of serum total cholesterol, different types of stroke, and stenosis distribution of cerebral arteries. The Akita Pathology Study. Stroke. (1993) 24:954-64. doi: 10.1161/01.STR.24.7.954

37. Niroumand S, Khajedaluee $M$, Khademrezaiyan $M$, Maryam A, Mohammadreza J, Gholamhasan K, et al. Atherogenic Index of Plasma (AIP): a marker of cardiovascular disease. Med J Islamic Republic of Iran. (2015) 29:240.

38. Hansson GK. Mechanisms of disease: inflammation, atherosclerosis, and coronary artery disease. Nature. (2005) 306:5.

39. Yu S, Arima H, Heeley E, Delcourt C, Krause M, Peng B, et al. White blood cell count and clinical outcomes after intracerebral hemorrhage: the INTERACT2 trial. J Neurol Sci. (2016) 361:112-6. doi: 10.1016/j.jns.2015. 12.033

40. Shoamanesh A, Preis SR, Beiser AS, Vasan RS, Benjamin EJ, Kase CS, et al. Inflammatory biomarkers, cerebral microbleeds, and small vessel disease: Framingham Heart Study. Neurology. (2015) 84:825-32. doi: 10.1212/WNL.00000000000 01279

Conflict of Interest: The authors declare that the research was conducted in the absence of any commercial or financial relationships that could be construed as a potential conflict of interest.

Copyright (C) 2019 Chen, Mo, Xu, Qin, Zheng, Pan, Meng, Jing, Xiang and Wang. This is an open-access article distributed under the terms of the Creative Commons Attribution License (CC BY). The use, distribution or reproduction in other forums is permitted, provided the original author(s) and the copyright owner(s) are credited and that the original publication in this journal is cited, in accordance with accepted academic practice. No use, distribution or reproduction is permitted which does not comply with these terms. 